\title{
Teses produzidas nos programas de Pós-Graduação em Enfermagem de 1983 a 2001
}

\author{
NURSING DOCTORAL THESES PRODUCED ON GRADUATE PROGRAMS BETWEEN 1983-2001
}

TESIS PRODUCIDAS EN LOS PROGRAMAS DE POSTGRADO EN ENFERMERÍA DE 1983-2001

\begin{abstract}
Alacoque Lorenzini Erdmann ${ }^{1}$, Isília Aparecida Silva ${ }^{2}$, Rosalina Aparecida Partezani Rodrigues ${ }^{3}$, Josicélia Dumêt Fernandes ${ }^{4}$, Lucila Amaral Carneiro Vianna ${ }^{5}$, Marta Júlia Marques Lopes ${ }^{6}$, Rosangela da Silva Santos ${ }^{7}$, Thelma Leite de Araújo ${ }^{8}$
\end{abstract}

\section{RESUMO}

Este estudo descreve a relação das teses de doutorado em enfermagem defendidas nos Programas de PósGraduação em Enfermagem, no Brasil, com as linhas de pesquisas definidas pela área da Enfermagem, em três campos: profissional, assistencial e organizacional. É uma pesquisa descritiva-exploratória, com base nos relatórios dos programas de Pós-Graduação, avaliados pela CAPES e no Catálogo Informações sobre Pesquisas e Pesquisadores em Enfermagem CEPEn/ ABEn, refe-rentes ao período de 1983-2001, totalizando 448 resumos. O campo assistencial alberga o maior número de produção, seguido do organizacional e do profissional. As investigações evidenciam uma tendência para estudos de abordagem qualitativa, descortinando possibilidades de aprofundamento do conhecimento da realidade ou compreensão de fenômenos sociais que tangenciam a prática da enfermagem, porém, os estudos de intervenção na prática profissional e de desenvolvimento de tecnologia ainda estão em fase de construção.

\section{DESCRITORES}

Pesquisa em enfermagem.

Educação de pós-graduação em enfermagem (tendências).

\section{ABSTRACT}

This study describes the relationship between nursing doctoral dissertations and research lines defined by the Brazilian Nursing field in three areas: professional, care and organizational. It isa descriptive-exploratory research, based on reports of Graduate Programs evaluated by CAPES and on The Informative Guide of Nursing Research and Researchers CEPEn/ ABEn, from 1983 to 2001 , totalling 448 abstracts. The care field includes the greatest production, followed by the organizational and professional areas. The studies disclose tendency towards the qualitative approach revealing possibilities for a more profound knowledge about reality or a more through understanding of social phenomena related to nursing practice. On the other hand, studies on interventions in professional practice and technological development are still under construction.

\section{KEY WORDS}

Nursing research.

Education, nursing, graduate (trends)

\section{RESUMEN}

Este estudio ofrece una descripción de la relación de tesis de doctorado en enfermería sustentadas en los Programas de Post Grado en Enfermería, en el Brasil, con las líneas de investigación definidas por el área de Enfermería, en tres campos: profesional, asistencial y organizacional. Es una investigación descriptiva-exploratoria, basada en los informes de los programas de PostGrado, evaluados por la CAPES y en el Catálogo Informaciones sobre Investigaciones e Investigadores en Enfermería CEPEn/ABEn, referentes al período de 1983-2001, totalizando 448 resúmenes. El campo asistencial alberga el mayor número de producción, seguido del organizacional y del profesional. Las investigaciones evidencian una tendencia hacia estudios de abordaje cualitativo, develando posibilidades de profundización del conocimiento de la realidad o comprensión de fenómenos sociales que tangencian la práctica de la enfermería, a pesar que los estudios de intervención en la práctica profesional y de desarrollo de la tecnología se encuentren, aún, en fase de construcción.

\section{DESCRIPTORES}

Investigación en enfermería. Educación de postgrado en enfermería (tendencias).
1 Professor Titular do Departamento de Enfermagem da Universidade Federal de Santa Catarina alacoque@newsite.com.br

2 Professor Titular da Escola de Enfermagem da Universidade de São Paulo

3 Professor Titular da Escola de Enfermagem de Ribeirão Preto da Universidade de São Paulo

4 Professor Titular do Departamento de Enfermagem da Universidade Federal da Bahia

5 Titular do Departamento de Enfermagem da Universidade Federal de São Paulo

6 Professor Titular da Escola de Enfermagem da Universidade Federal do Rio Grande do Sul

7 Professor Titular da escola de Enfermagem Anna Nery da Universidade Federal do Rio de Janeiro

8 Professor Adjunto do Departamento de Enfermagem da Universidade Federal do Ceará 
Alacoque L. Erdmann Isília Aparecida Silva

Rosalina A. P. Rodrigues Josicélia $D$. Fernandes Lucila Amaral C. Vianna Marta J. Marques Lopes Rosangela S. Santos Thelma Leite de Araújo

\section{INTRODUÇÃO}

O avanço no desenvolvimento científico e tecnológico exige uma ampla abertura para a diversidade e interdisciplinaridade de conhecimentos, assim como um ambiente de investigação que propicie as relações e interações dos atores sociais, centrados na construção de saberes e práticas que resultem em novas tecnologias e novos conhecimentos para o melhor viver humano.

Nesse panorama, a Pós-Graduação (PG) se constitui num espaço de prática que possibilita o desenvolvimento de pesquisadores, das ações investigativas, dos processos de construção de conhecimentos e de novas tecnologias, onde as linhas de pesquisa se constituem em um dos principais eixos norteadores dessas atividades.

A Pós-Graduação brasileira vem crescendo, de forma significativa, nos últimos anos, adquirindo uma posição de destaque no sistema de ensino superior do País.

Os Programas de Pós-Graduação, vinculados às universidades públicas e privadas, adquirem validade nacional após serem recomendados pelo Ministério da Educação/Coordenadoria de Aperfeiçoamento de Pessoal do Ensino Superior, MEC/CAPES, e acompanhados através de um processo de avaliação continuada. Este processo, por sua vez, busca reforçar as potencialidades, apontar fragilidades e distorções de cada Programa, além de implementar diretrizes que possam promover a qualidade da Pós-Graduação, rumo à excelência acadêmica na formação de mestres e doutores.

A Área da Enfermagem, fazendo parte do sistema de Pós-Graduação brasileiro, vem sendo avaliada pelo Ministério da Educação/CAPES, desde os anos 70. A partir de 1993, a Área da Enfermagem passou a fazer parte da Grande Área da Saúde, constituída, atualmente, pelas áreas da Saúde Coletiva, Odontologia, Farmácia, Medicina I, II e III, Educação Física e Enfermagem.

Até 2003, a Área da Enfermagem contava com 21 Programas de Pós-Graduação stricto-sensu reconhecidos, assim distribuídos: 4 na Região Nordeste, 12 na Sudeste, 4 na Sul e um na Centro-Oeste. A Região Norte não possui, ainda, Programas de Pós-Graduação na área da Enfermagem ${ }^{(1)}$.

O crescimento dos cursos de doutorado, no Brasil, nas suas mais diversas áreas, no período de 1995 a 2001, foi de 25\%, ou seja, de um total de 685 cursos, em 1995, passou, em 2001, para 857, e o número de alunos matriculados passou, nesse mesmo período, de 19.492 para 32.731, com um crescimento de $67,9 \%$. A evolução em relação ao número de doutores titulados foi mais expressiva ainda, com $141,9 \%$ de crescimento, ou seja, de 2.497 doutores titulados em 1995 passou para $6.042 \mathrm{em} 2001^{(2)}$.

Do total dos cursos de doutorado do País, 25\% são da área da saúde ${ }^{(3-4)}$, o que indica ser uma área bastante expressiva frente às demais. Nessa área estão incluídos os dez Programas de Pós-Graduação em Enfermagem que oferecem os Cursos de Doutorado, sendo um na Região Nordeste, 8 na Sudeste e um na Região Sul.

A formação de doutores em enfermagem se inicia com a criação do primeiro curso, em 1981, o Programa Interunidades de Doutoramento em Enfermagem da Universidade de São Paulo, seguido dos programas denominados hoje de Pós-Graduação em Enfermagem da Universidade Federal de São Paulo, criado em 1986, e da Escola de Enfermagem da Universidade de São Paulo, em 1989. Os demais cursos de doutorado surgiram na década de 90, exceto o de Enfermagem na Saúde do Adulto, da escola de Enfermagem da USP, criado em 2002.

O processo de formação de doutores em enfermagem situa-se na capacitação de profissionais para o desenvolvimento da pesquisa, do ensino e da atenção à saúde da população. Nesse processo, a construção de novos conhecimentos da área se constitui em elemento importante para o desenvolvimento científico e tecnológico da enfermagem e da saúde, tendo as linhas de pesquisa como eixo norteador desse processo.

Na tentativa de conhecer o eixo norteador das ações investigativas dos cursos de doutorado em enfermagem, no Brasil, o presente estudo busca identificar as teses defendidas nos programas de pós-graduação em enfermagem, no Brasil, e suas respectivas linhas de pesquisas, tendo como referência as linhas de pesquisa definidas pela Área da Enfermagem/CAPES, em 2001(5-6).

Parte-se do pressuposto de que conhecendo melhor o eixo condutor do que é produzido pelos cursos de doutorado em Enfermagem, através de suas teses, é possível obter subsídios para o estabelecimento ou re-direcionamento de políticas de formação de doutores e de planejamento e fomento de programas de pesquisa. Isto favorece o avanço no desenvolvimento científico e tecnológico das áreas de Enfermagem e da Saúde, bem como, abre possibilidades para a construção de padrões científicos compatíveis com as necessidades da excelência acadêmica.
Rev Esc Enferm USP 2005; 39(Esp.):497-505. 


\section{METODOLOGIA}

Este estudo, do tipo descritivo-exploratório, foi realizado mediante o levantamento dos resumos das teses dos Programas de Pós-Graduação em Enfermagem, nível de doutorado, junto aos dados disponíveis nos relatórios de avaliação do MEC/CAPES e às publicações da Associação Brasileira de Enfermagem - CEPEn/ABEn - Informações Sobre Pesquisas e Pesquisadores do Centro de Estudos e Pesquisas em Enfermagem - disponíveis em CD (compact disk), referentes ao período de 1983 a 2001.

Embora os catálogos já sejam publicados desde 1979, optou-se por incluir, no presente estudo, as teses dos programas de Pós-Graduação em Enfermagem a partir de 1983, ano em que foi publicado o resumo da primeira tese produzida.

A coleta de dados foi realizada mediante a aplicação de um instrumento de registro, que constou de itens como: local e ano de defesa da tese, instituição e/ou programa de Pós-Graduação, título da tese, além dos dados específicos sobre as características temáticas e metodológicas das teses analisadas por meio dos resumos disponibilizados.
É importante ressaltar, que foram analisados os resumos disponibilizados nas fontes anteriormente mencionadas, o que não totaliza o número de teses defendidas em programas de Pós-Graduação, nem tampouco teses defendidas por enfermeiros em outros programas, ainda, que a primeira tese defendida em programa de Pós-Graduação em Enfermagem data de 1983.

Para análise dos dados, utilizou-se as linhas de pesquisas definidas pela Área da Enfermagem/ CAPES, em 2001 ${ }^{(5-6)}$, agrupadas em três campos de ação, ou seja, área profissional, assistencial e organizacional, de acordo com o Quadro 1.

De uma maneira geral, as linhas de pesquisa existentes nos dez Programas de Pós-Graduação são pertinentes às linhas estabelecidas como o proposto pela Área da Enfermagem/CAPES. Estas abrangem temas e projetos relacionados ao campo de conhecimento da Enfermagem, delineando com maior clareza os enfoques e abrangência dos estudos para a área, o que possibilita que os programas de pós-graduação sejam mais produtivos e tenham linhas de pesquisa consolidadas. Verifica-se também, as tendências e o surgimento de novas temáticas e avanços epistemológicos, metodológicos e mesmo ontológicos, já que o conhecimento construído resulta de um processo realizado por, com e para seres humanos.
Teses produzidas nos programas de Pós-Graduação em Enfermagem de 1983 a 2001

Quadro 1 - Descrição das Linhas de Pesquisa na Área de Pós-Graduação da Enfermagem/CAPES Belém, 2001 ${ }^{(6)}$.

\begin{tabular}{|l|l|l|}
\hline Área/Campo 1 - Profissional & Área/Campo 2 - Assistencial & Área/Campo 3 - Organizacional \\
\hline $\begin{array}{l}\text { 1.1 Fundamentos teórico-filosóficos } \\
\text { do cuidar em Saúde e Enfermagem }\end{array}$ & $\begin{array}{l}\text { 2.1 Processo de cuidar em Saúde e } \\
\text { Enfermagem }\end{array}$ & $\begin{array}{l}\text { 3.1 Políticas e práticas em Saúde e } \\
\text { Enfermagem }\end{array}$ \\
\hline $\begin{array}{l}\text { 1.2 Tecnologia em Saúde e } \\
\text { Enfermagem }\end{array}$ & 2.2 Saúde e qualidade de vida & $\begin{array}{l}3.2 \text { Políticas e práticas de } \\
\text { Educação e Enfermagem }\end{array}$ \\
\hline 1.3 Ética em Saúde e Enfermagem & - & $\begin{array}{l}\text { 3.3 Produção social e trabalho em } \\
\text { Saúde e Enfermagem }\end{array}$ \\
\hline 1.4 História da Enfermagem & - & $\begin{array}{l}\text { 3.4 Gerenciamento dos serviços de } \\
\text { Saúde e de Enfermagem }\end{array}$ \\
\hline & - & $\begin{array}{l}3.5 \text { Informação/comunicação em } \\
\text { Saúde e Enfermagem }\end{array}$ \\
\hline
\end{tabular}

\section{RESULTADOS}

Foi analisado um total de 448 resumos de teses do período de 1983 a 2001. Os resumos analisados foram obtidos de teses defendidas nos oito Programas de Pós-Graduação de Enfermagem, entre os 10 que mantinham curso em nível de doutorado até a época de realização da pesquisa, e que tiveram re- sumos de teses publicados nos catálogos até 2001, a saber: Programa Interunidades de Doutoramento em Enfermagem (EEUSP/EERP-USP, criado em 1981), Curso de Doutorado em Enfermagem do Programa de Pós-Graduação em Enfermagem (UNIFESP 1986), Curso de Doutorado em Enfermagem do Programa de Pós-Graduação em Enfermagem (EEUSP 1989), Curso de Doutorado em Enfermagem do Pro- 
Alacoque L. Erdmann Isília Aparecida Silva Rosalina A. P. Rodrigues Josicélia D. Fernandes Lucila Amaral C. Vianna Marta J. Marques Lopes Rosangela S. Santos Thelma Leite de Araújo grama de Pós-Graduação em Enfermagem (EEAN/ UFRJ - 1990), Curso de Doutorado em Enfermagem Fundamental do Programa de Pós-Graduação em Enfermagem Fundamental (EERP/USP - 1991), Curso de Doutorado em Enfermagem do Programa de Pós-Graduação em Enfermagem (UFSC - 1993), Curso de Doutorado em Enfermagem do Programa de Pós-Graduação em Enfermagem (UFC - 1998), Curso de Doutorado em Enfermagem em Saúde Pública do Programa de Pós-Graduação em Enfermagem em Saúde Pública (EERP/USP - 1998). Vale salientar, que os cursos de doutorado dos Programas de PósGraduação em Enfermagem Psiquiátrica (EERP/USP) e em Enfermagem na Saúde do Adulto (PROESA/ EEUSP/USP), iniciaram seu funcionamento em 1999 e 2002, respectivamente, não sendo incluídos nesse estudo.

A maior parte dos resumos das teses, consultados até 2001, 318 (70,98\%), foi da Universidade de São Paulo, concluídas nos programas da EEUSP e EERP-USP. Nos parece que esta concentração de teses está relacionada ao fato destas duas instituições terem sido as primeiras a oferecerem os cursos de doutorado na Área da Enfermagem, e também, serem as únicas que possui mais de um programa de pós-graduação stricto-sensu na área, ou seja, atualmente contam com 6 Programas de Pós-Graduação em Enfermagem, todos ofertando o curso de Doutorado. Os demais resumos foram originários de teses defendidas nas seguintes instituições: UFRJ 72 (16,07\%), UFSC - 34 (7,58\%), UNIFESP - 21 (4,68\%), e UFC - 3 (0,66\%). Constata-se, portanto, que a maior produção está na Região Sudeste, onde se encontra a maioria dos Programas de Pós-Graduação em Enfermagem.

Da leitura dos 448 resumos de teses, identificou-se, através das temáticas de estudo e objetivos, a que linha de pesquisa estas produções se vinculam, tomando como referência as apresentadas no Quadro 1. Assim, os resultados obtidos mostram que foram produzidas mais teses nas áreas/ campos assistencial (171 ou 38,1\%) e organi-zacional (164 ou 36,6\%) do que na profissional (86 ou 19,1\%). Ressalta-se que 27 resumos não puderam ser classificados, nem mesmo por aproximação.

Destacam-se as linhas de pesquisa que apareceram em maior freqüência: Saúde e qualidade de vida, Processo de cuidar em Saúde e Enfermagem, do campo Assistencial; Políticas e práticas de Educação e Enfermagem, Gerenciamento dos Serviços da Saúde e de Enfermagem, do campo Organizacional; Fundamentos teórico-filosóficos do Cuidar em Saúde e Enfermagem, do campo Profissional, conforme descritas a seguir:

Tabela 1 - Distribuição dos resumos das teses da Pós-graduação em Enfermagem, nível doutorado, apresentadas nos relatórios da CAPES/MEC e as publicadas no catálogo CEPEn, de 1987 a 2001 , segundo as linhas de pesquisas da CAPES - São Paulo, 2003.

\begin{tabular}{|c|c|c|}
\hline Área/campo: Profissional & $\mathbf{N}$ & $\%$ \\
\hline Fund. teórico-filosóficos do cuidar em Saúde e Enfermagem & 51 & 11,38 \\
\hline Tecnologia em Saúde e Enfermagem & 17 & 3,79 \\
\hline Ética em Saúde e Enfermagem & 7 & 1,56 \\
\hline História da Enfermagem & 11 & 2,45 \\
\hline Sub-total 1 & 86 & 19,19 \\
\hline \multicolumn{3}{|l|}{ Área/campo: Assistencial } \\
\hline Proceso de cuidar em Saúde e Enfermagem & 80 & 17,85 \\
\hline Saúde e qualidade de vida & 91 & 20,31 \\
\hline Sub-total 2 & 171 & 38,16 \\
\hline \multicolumn{3}{|l|}{ Área/campo: Organizacional } \\
\hline Políticas e práticas em Saúde e Enfermagem & 13 & 2,90 \\
\hline Políticas e práticas de Educação e Enfermagem & 57 & 12,72 \\
\hline Produção social e trabalho em Saúde e Enfermagem & 34 & 7,58 \\
\hline Gerenciamento dos serviços de Saúde e de Enfermagem & 43 & 9,59 \\
\hline Informação/comunicação em Saúde e Enfermagem & 17 & 3,79 \\
\hline Sub-total 3 & 164 & 36,60 \\
\hline Área/campo: não identificado & 27 & 6,02 \\
\hline Total & 448 & 100,00 \\
\hline
\end{tabular}


A Tabela 1 revela que os objetos de pesquisa relacionados ao campo assistencial mantêm a liderança quando comparados com o cenário de investigação científica na enfermagem, nas décadas de $50-70^{(7)}$ e $80-90^{(8)}$. Embora os estudos sobre a produção de pesquisas nesse período, não adotem a mesma classificação atual das linhas de pesquisas MEC/CAPES, ao se considerar os resultados destes e de outros mais recentes ${ }^{(9-10)}$, é possível perceber que as décadas de 50 a 90 foram marcadas pelo interesse no desenvolvimento de estudos que subsidiassem a assistência de enfermagem, refletindo no aumento de pesquisas nessa área, subordinadas ao modelo biomédico e de caráter hospitalar, em que predominavam investigações cujos objetos versavam sobre a avaliação da assistência, necessidades dos pacientes e os processos assistenciais. Em menor número, os estudos sobre papéis, funções e atribuições da enfermagem, bem como, o processo de trabalho e necessidades sentidas pela equipe, e ensino com ênfase na formação profissional, nos anos 50, tiveram forte influência dos movimentos a favor da implantação dos cursos de enfermagem no nível universitário. Da mesma forma, pesquisas realizadas e publicadas sobre esse mesmo tema, continuam ocupando destaque nas décadas seguintes, ainda aquém da área assistencial, motivados pela inserção da enfermagem nos sistema de pós-graduação e definição do currículo mínimo para os cursos de graduação, sendo esta abordagem temática ainda a que prevalece dentro do campo organizacional, segundo a classificação atual de linhas de pesquisa.

Em terceiro lugar, identificamos o campo profissional, o qual teve grande avanço nas décadas de 1950 a 1990, em especial com estudos classificados como "metodologia de enfermagem"(7-8), voltados em sua maioria, para o desenvolvimento dos métodos ou técnicas de assistência de enfermagem. Observa-se aqui, como em outros trabalhos, que o campo profissional ainda é o que abarca o menor número de pesquisas ${ }^{(9)}$. Porém, percebe-se pelo presente estudo que o enfoque predominante atualmente, refere-se à construção de bases teóricas para o cuidado, em detrimento das bases técnicas. Ainda, que dentro do campo profissional, há um pequeno número de pesquisas em História da Enfermagem, o que se justifica pelo fato de serem poucos os programas de Pós-Graduação que adotam linhas específicas que aprofundam esse tema. Da mesma forma, as pesquisas voltadas para as questões da Ética e Bioética também são em menor número, pelo fato desta temática ser mais recente e, em alguns programas, as linhas a que estes estudos estão vinculados, serem consideradas, ainda, como emergentes. Ainda, a linha de pesquisa "Informação/Comunicação em Saúde e Enfermagem” apresenta-se em crescimento na última década, por abranger temas que despertaram o interesse dos enfermeiros a partir da era da informática.

Quanto à identificação do método de investigação ou tipo de estudo ou pesquisa, verificou-se que, apesar da maioria dos resumos não explicitar com devida clareza qual o método de pesquisa utilizado, foi possível agrupá-los, tomando-se em conta também o referencial teórico e o método de análise explicitado.

Fica evidente, de certo modo, que prevalecem os estudos exploratórios, de abordagem qualitativa, de conhecimento da realidade ou compreensão dos fenômenos sociais. Os estudos de intervenção na prática profissional ou de desenvolvimento de tecnologias, ainda estão aquém das necessidades da prática profissional.

A distribuição das teses, segundo o método de investigação pode ser observado na Tabela 2. Do total, verifica-se que 212(47,32\%) foram estudos qualitativos, desenvolvidos a partir de métodos como a fenomenologia, 41 (19,33\%), estudos de representações sociais, 21 (9,90\%), pesquisa histórica, 18 (8,49\%), estudos etnográficos, 17 (8,01\%), teoria fundamentada em dados, 17 (8,01\%), materialismo histórico e dialético 14 (6,60\%), hermenêutica dialética, seis (2,83\%), pesquisa participante, cinco (2,35\%), dentre outros. Esses dados refletem a tendência crescente de estudos qualitativos que teve início em final da década de 80 e que demarcam a transição de uma perspectiva de investigação científica na ótica positivista, que, por sua vez, permitia uma visão parcial dos processos vivenciais ${ }^{(11-12)}$.
Teses produzidas nos programas de Pós-Graduação em Enfermagem de 1983 a 2001 
Alacoque L. Erdmann Isília Aparecida Silva Rosalina A. P. Rodrigues Josicélia $D$. Fernandes Lucila Amaral C. Vianna Marta J. Marques Lopes Rosangela S. Santos Thelma Leite de Araújo

Tabela 2 - Distribuição das teses dos programas de Pós-Graduação em Enfermagem de 1983 a 2001 , segundo o método de investigação - São Paulo, 2003.

\begin{tabular}{lrr}
\hline Método de investigação & N & $\%$ \\
\hline Não explicitou ou não foi possível identificar & 85 & 18,97 \\
Estudo qualitativo & 212 & 47,32 \\
Estudo quantitativo & 104 & 23,21 \\
Quali-quantitativo & 5 & 1,11 \\
Estudo exploratório-descritivo & 20 & 4,46 \\
Estudo de caso & 12 & 2,67 \\
Estudo epidemiológico social/epidemiológico & 10 & 2,32 \\
\hline Total & 448 & $\mathbf{1 0 0 , 0 0}$
\end{tabular}

A adoção dos métodos qualitativos reflete a mudança de paradigmas em busca da totalidade do fenômeno e aproximação mais ampla da realidade ${ }^{(11)}$. Assim, o incremento de pesquisas qualitativas ocorreu na maioria dos programas de Pós-Graduação, abrangendo grande variedade de temas estudados pela enfermagem, tornando-se a principal abordagem de pesquisa na segunda metade da década de 1990 utilizada nas teses estudas ${ }^{(11-12)}$.

Essa tendência pode explicar, mesmo que parcialmente, a razão pela qual os objetos de estudo que versam sobre a compreensão da realidade têm demonstrado ser importantes para o avanço e consolidação do conhecimento na área, evidenciando uma concentração de pesquisas em linhas como Fundamentos Teórico-Filosóficos do Cuidar em Saúde e Enfermagem.

Os estudos quantitativos, por sua vez, totalizaram 104 (23,21\%) e foram desenvolvidos a partir de modelos clássicos da pesquisa como o survey, 22 (21,15\%), descritivos experimentais/semi-experimentais 15 (14,42\%), testagem ou validação de instrumentos 15 (14,42\%) e estudos exploratórios 15 $(14,42 \%)$, dentre outros.

Assim, a transição da hegemonia dos métodos quantitativos para adoção dos qualitativos, demonstra o interesse em objetos de estudos que resultam em produção que avança o domínio da prática de enfermagem para uma construção teórica mais ampla para o setor saúde.

Outro aspecto a ser considerado é a realização de estudos que combinam os métodos quali e quantitativo, 5 (1,11\%), demonstrando uma tendência sem, contudo, demonstrar uma clareza da finalidade de triangulação para o domínio mais amplo e mais aprofundado do conhecimento sobre o objeto de estudo.

A diversidade de adoção de métodos de pesquisa, com aprofundamento de bases teóricas e filosó- ficas, demonstra uma apropriação do conhecimento sobre essas bases, incutindo a aplicação de perspectivas de outras disciplinas para o estudo dos fenômenos da enfermagem ${ }^{(12-13)}$.

No entanto, é necessário ressaltar que a qualidade dos resumos não permitiu avaliar os métodos utilizados para 85 (18,97\%) destes. Esse fato nos leva a inferir que há, por parte dos autores, uma desconsideração ou desconhecimento sobre a estrutura do resumo, como fonte de informação sobre os principais elementos da pesquisa e, também, equívocos sobre a conceituação de métodos e técnicas de coleta de dados.

A identificação das áreas temáticas mais estudadas nas teses da Enfermagem mostra uma convergência de interesse para o tema Saúde da MuIher que teve 67 (14,95\%) teses e para o Gerenciamento em Enfermagem e Saúde com 66 (14,73\%). A seguir, os temas mais estudados foram os relativos a Saúde Coletiva 45 (10,04\%) e Saúde da Criança 43 (9,54\%). A predominância destes temas pode estar relacionada ao fato das primeiras linhas de pesquisa adotadas nos Programas de Pós-Graduação, criadas a partir de Áreas de Concentração em Saúde da Mulher, Saúde da Criança, Saúde Coletiva/Saúde Pública e Administração/Gerenciamento, desde a década de 70, nos primórdios do ensino de PósGraduação. Da mesma forma, é possível encontrar áreas de concentração ou pelo menos linhas de pesquisa, que enfoquem de alguma forma essas temáticas, em quase a totalidade dos atuais programas de Pós-Graduação da área da Enfermagem ${ }^{(14)}$.

Por outro lado, novas temáticas emergiram ao longo das últimas décadas. Os demais temas desenvolvidos, aqui considerados na categoria "Outras”, 75 (16,74\%), somam os estudos na área da saúde do trabalhador, saúde da família, entre outros. As primeiras pesquisas nessas temáticas, embora, tenham sido desenvolvidos no final da década de 1980, só passaram a ter uma regularidade de
Rev Esc Enferm USP 2005; 39(Esp.):497-505. 
teses defendidas a partir de meados da década de 1990. É possível que o número de trabalhos desenvolvidos nessas áreas, seja superior ao aqui constatado nos catálogos do CEPEn/ABEn, por terem seus objetos de pesquisa definidos na interface com outras áreas como Saúde Coletiva, Saúde da Crian- ça e Gerenciamento de Serviços de Enfermagem, pelo fato dos autores terem adotado uma terminologia que não permite a visualização do estudo na perspectiva de uma construção temática própria. Esses resultados estão ilustrados na Tabela 3.
Teses produzidas nos programas de Pós-Graduação em Enfermagem de 1983 a 2001

Tabela 3 - Distribuição dos resumos das teses dos programas de Pós Graduação em Enfermagem de 1983 a 2001, segundo as áreas temáticas - São Paulo, 2003.

\begin{tabular}{lcc}
\hline Áreas Temáticas & N & $\%$ \\
\hline Outras* & 75 & 16,74 \\
Saúde da mulher & 67 & 14,95 \\
Gerenciamento em enfermagem e saúde & 66 & 14,73 \\
Saúde coletiva & 45 & 10,04 \\
Saúde da criança & 43 & 9,59 \\
Saúde do adulto & 33 & 7,36 \\
Educação & 33 & 7,36 \\
Saúde Mental & 23 & 5,13 \\
Tecnologia em enfermagem & 20 & 4,46 \\
Informação e comunicação & 15 & 3,34 \\
Saúde do idoso & 11 & 2,45 \\
História da enfermagem & 11 & 2,45 \\
Ética & 6 & 1,33 \\
\hline Total & 448 & $\mathbf{1 0 0 , 0 0}$ \\
\hline
\end{tabular}

*Trabalho em Enfermagem, Saúde do trabalhador, Saúde da família, Pesquisa em Enfermagem, Exercício físico, Fisiologia, Trauma, Infecções, Processo saúde e doença, Morte.

Chama a atenção os 20 (4,46\%) estudos em Tecnologia em enfermagem, realizados na sua maioria, com pesquisas experimentais, semi-experimentais, testagens ou validações de instrumentos, modelos ou processos que, embora em número pouco expressivo, se encontram presentes nas principais temáticas estudadas.

\section{CONSIDERAÇÕES FINAIS}

A análise da produção científica originada das teses estudadas, nos indica a legitimidade das linhas de pesquisa definidas pela Área de Enfermagem/CAPES desde 2001, no que concerne à capacidade de abrangência dessas para a produção da enfermagem, possibilitando acompanhar as tendências de construção do conhecimento na área.

Os resultados do presente estudo demonstram que, embora as teses aqui analisadas, tenham sido concluídas em período anterior à definição das referidas linhas de pesquisa, a maioria delas se insere nas linhas propostas, refletindo a legitimidade das mesmas e a coerência dessas com a atividade de pesquisa desenvolvida na área da Enfermagem. Isto significa que são linhas de pesquisa consolidadas e consistentes. Um pequeno percentual de teses não foi inserido nas linhas pelo fato de não ter sido possível a identificação de elementos que possibilitasse sua classificação.
Assim, observa-se que a produção do conhecimento, a partir das teses, ocorreu com maior freqüência no campo Assistencial, indicando uma concentração de estudos em suas duas linhas de pesquisa, que supera numericamente as demais linhas dos outros dois campos, o que sugere uma tendência importante para a investigação científica na enfermagem. A prevalência de pesquisas nessa área mantém uma continuidade há quatro décadas, indicando, no entanto, uma mudança de ótica das questões relativas aos objetos de estudo, induzindo a adoção de novos métodos de pesquisa.

Quanto ao campo Organizacional, o qual abarca o segundo maior número de teses avaliadas, entre suas cinco linhas, destaca-se a linha de pesquisa "Políticas e práticas de educação em enfermagem” e a de "Gerenciamento em serviço de saúde e de enfermagem”, o que, a exemplo do comentário anterior, também, demonstra a mesma tendência de continuidade de interesse dos enfermeiros em pesquisar temas dentro desse assunto, provavelmente motivados pelos contextos de contínua mudança nas estruturas organizacionais da enfermagem, seja na dimensão do ensino formal como da administração dos serviços de saúde.

E, no campo Profissional, destaca-se a linha "Fundamentos teórico filosóficos do cuidar em saúde em enfermagem”, que sobressai significativamente sobre as três demais, o que nos leva a inferir so-

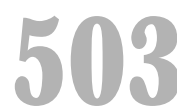

Rev Esc Enferm USP 2005; 39(Esp.):497-505. 
Alacoque L. Erdmann Isília Aparecida Silva Rosalina A. P. Rodrigues Josicélia $D$. Fernandes Lucila Amaral C. Vianna Marta J. Marques Lopes Rosangela S. Santos Thelma Leite de Araújo bre a influência dos programas de Pós-Graduação mais diretamente sobre esse tipo de estudo, uma vez que foi no ambiente acadêmico que se iniciou e se mantém a melhor condição para suscitar e sustentar estudos dessa natureza.

Da mesma forma, esses estudos nos indicam uma mudança de paradigma no processo de construção de conhecimento na enfermagem, evidenciando um direcionamento para os estudos com abordagem qualitativa. Nestes, os estudos fenomenológicoshermenêuticos, etnográficos, históricos e sociais, dentre outros, descortinam possibilidades de aprofundamento do conhecimento da realidade ou compreensão dos fenômenos sociais que permeiam a prática da enfermagem. A adoção de diversos métodos e abordagens teóricas tem exigido a apropriação de conteúdos e fundamentação provinda de outras disciplinas, o que provoca uma expansão de possibilidades para a construção do conhecimento e aprimoramento do profissional do ponto de vista científico e cultural.

Os resultados evidenciaram, também, que os estudos de intervenção na prática profissional e de desenvolvimento de tecnologia ainda são poucos e demonstram tendência de construção, o que deve ser sinalizado aos pesquisadores a necessidade urgente deste tipo de pesquisa para ser aplicada à prática profissional.

Se, por um lado, houve um avanço na compreensão de fenômenos sociais, por outro, pode ser o reflexo de uma necessidade de se olhar os objetos de estudos provenientes da prática sob outra ótica,

\section{REFERÊNCIAS}

(1) Coordenação de Aperfeiçoamento de Pessoal de Nível Superior (CAPES). Linhas de ação e programas. Relatório de transição da CAPES. Brasília; 2002.

(2) Matos BP, Velloso J. Contexto e objetivos. In: Jacques Velloso, organizador. Formação no país ou no exterior? Doutores na pós-graduação de excelência. Brasília; 2002. p. 37-44.

(3) Balbachevsky E, Velloso J. Atividades editoriais, comitês e trajetórias profissionais. In: Jacques Velloso, organizador. Formação no país ou no exterior? Doutores na pós-graduação de excelência. Brasília; 2002. p. 201-16.

(4) Coordenação de Aperfeiçoamento de Pessoal de Nível Superior (CAPES). Sistema de Avaliação. Coleta de Dados 6.0: Manual do usuário. Brasília; 2001.

(5) Associação Brasileira de Enfermagem. Consolidação das propostas de linhas de pesquisa em enfermagem. [online] Brasília; 2001. Disponível em: <http://www. abennacional.org.br/documentos/linha pesq 11senpe. doc $>$ ( 08 jun. 2005) não havendo, necessariamente, mudança de objetos, mas sim de perspectivas de compreensão dos mesmos. Em um primeiro momento da atividade de pesquisa, há necessidade de construir uma prática pautada na ciência, e remeter os estudos de técnicas e modelos assistenciais voltados para o cotidiano do fazer.

No que diz respeito à legitimidade das linhas de pesquisas da Área de Enfermagem na CAPES, mesmo aquelas que apresentam um número reduzido de pesquisas como Ética em Saúde e Enfermagem e História da Enfermagem, não significa que não sejam consolidadas. Isto apenas é o reflexo de uma valorização mais recente desses temas, pelo interesse social que os mesmos têm despertado a partir da última década.

A necessidade da produção de conhecimentos na Enfermagem para a visibilidade nacional e internacional, o reconhecimento e a consolidação da Pós-Graduação, da Graduação e da Profissão requer o avanço científico e tecnológico da profissão que, por sua vez, requer pesquisadores competentes e uma ampla política nacional de pesquisa.

A internacionalização da Enfermagem brasileira é fruto das fortalezas regionais e a soma de esforços regionais, a determinação para alcançar metas. O prazer da descoberta, da criação, da construção de conhecimentos, tem sido uma prática social desafiadora e a Pós-Graduação tem papel fundamental no avanço do conhecimento e por sua vez, sua aplicabilidade na prática profissional.
Rev Esc Enferm USP 2005; 39(Esp.):497-505.
(6) Carvalho V. Linhas de pesquisa e prioridades de enfermagem: proposta com distinção gnoseológica para o agrupamento da produção científica de pós-graduação em enfermagem. Esc Anna Nery Rev Enferm. 2002; 6(1):145-54.

(7) Nogueira MJC. A pesquisa em enfermagem no Brasil: retrospectiva histórica. Rev Esc Enferm USP 1982; 16(1):17-26.

(8) Cianciarullo TI, Salzano SDT. A enfermagem e a pesquisa no Brasil. Rev Esc Enferm USP 1991; 25(2): 195-215.

(9) Rodrigues RAP, Erdmann AL, Silva IA, Fernandes JD, Santos RSS, Araújo TL. Formação de doutores em enfermagem no Brasil. Texto \& Contexto Enferm 2002; 11(2):66-76.

(10) Silva MJP, Ângelo M, Castilho V, Egry EY, Rocha SM, Trzeniak P. Revista da escola de enfermagem da universidade de São Paulo: 36 anos zelando pela qualidade do trabalho na Enfermagem. Rev Esc Enferm USP 2004; 38(1):109-13. 
(11) Ray MAA. A philosophical: a method for nursing research. Nurs Sci 1983; (52)49-63.

(12) Silva IA, Oliveira MA. Qualitative research in Brazil: state of the art and tendencies. In: Program of the $2^{\text {th }}$ Advances in Qualitative Methods Conference; 2001 Feb. 22-24; Edmonton (CAN). Edmonton: International Institute Qualitative Methododoly/ University of Alberta; 2001. p. 84.
(13) Silva IA, Ferriani MGC. Teses defendidas por alunos do Programa Interunidades de Doutoramento em Enfermagem: a parcela da construção do conhecimento para a assistência, ensino e pesquisa. Rev Esc Enferm USP. No prelo 2006.

(14) Coordenação de Aperfeiçoamento de Pessoal de Nível Superior (CAPES). Cadernos de avaliação. [online] Brasília; 2004. Disponível em:http:// www1.capes.gov.br/Scripts/Serviços/Indicadores/ Dados/SelecionaAno.idc (14 fev. 2005)
Teses produzidas nos programas de Pós-Graduação em Enfermagem de 1983 a 2001
Correspondência: Alacoque L. Erdmann Univ. Fed. de Sta. Catarina Centro de Saúde

- Dept ${ }^{\circ}$ de Enfermagem - Trindade - Florianópolis CEP - 88040-900 - SC 